\title{
Yang-Mills theory for nonsemisimple groups
}

\author{
Jean Nuyts* \\ Université de Mons-Hainaut, 20 Place du Parc, 7000 Mons, Belgium \\ Tai Tsun $\mathrm{Wu}^{\dagger}$ \\ Gordon McKay Laboratory, Harvard University, Cambridge, Massachusetts 02138 \\ and Theoretical Physics Division, CERN, CH-1211 Geneva 23, Switzerland
}

(Received 21 October 2002; published 27 January 2003)

\begin{abstract}
For semisimple groups, possibly multiplied by U(1)'s, the number of Yang-Mills gauge fields is equal to the number of generators of the group. In this paper, it is shown that, for nonsemisimple groups, the number of Yang-Mills fields can be larger. These additional Yang-Mills fields are not irrelevant because they appear in the gauge transformations of the original Yang-Mills fields. Such nonsemisimple Yang-Mills theories may lead to physical consequences worth studying. The nonsemisimple group with only two generators that do not commute is studied in detail.
\end{abstract}

DOI: 10.1103/PhysRevD.67.025014

PACS number(s): 11.15.- q, 11.10.-z

\section{INTRODUCTION}

The non-Abelian gauge field theory was invented by Yang and Mills [1] almost half a century ago; it now permeates the study of elementary particles, both strong and electroweak. In all cases, the number of spin-1 gauge fields is equal to the number of generators of the gauge group. For example, for the SU(2) case, there are three generators and also three Yang-Mills fields. It is the purpose of the present paper to study the question: What happens if more Yang-Mills fields are introduced than the number of generators of the gauge group?

This possibility has actually been investigated already in the original paper of Yang and Mills [1]. They showed that such additional gauge fields are "allowed by the very general form" but "irrelevant to the question of isotopic gauge."

It is this sentence of Yang and Mills that initiated the present investigation. Since their conclusion holds whenever the gauge group is semisimple, the additional gauge fields are of interest for nonsemisimple gauge groups. It is found that, at least for some cases and perhaps in most cases, the number of relevant spin-1 gauge fields can be larger than the number of generators of the underlying gauge group. It is hoped that this phenomenon, which we did not anticipate, may be of use for particle physics.

In particle physics, the "simplest" Lie groups seem to play the most fundamental roles. For example, in the electroweak theory of Glashow [2], Weinberg [3] and Salam [4], the group is $\mathrm{SU}(2) \otimes \mathrm{U}(1)$. It is therefore the purpose here to study the "simplest" nonsemisimple group [other than $\left.\mathrm{U}(1)^{n}\right]$ in the sense that the number of generators is the smallest. If there is only one generator, then the Lie group is necessarily Abelian. We shall concentrate here on the nonAbelian Lie group with two generators.

These two generators $L_{1}$ and $L_{2}$ can be chosen to obey the commutation relation

\footnotetext{
*Email address: Jean.Nuyts@umh.ac.be

${ }^{\dagger}$ Email address: ttwu@deas.harvard.edu
}

$$
\left[L_{1}, L_{2}\right]=L_{2}
$$

This commutation relation leads essentially to only one gauge group. Faithful representations of the lowest dimension are

$$
\begin{aligned}
& L_{1}=\left(\begin{array}{cc}
\frac{1}{2}+s & 0 \\
0 & -\frac{1}{2}+s
\end{array}\right), \\
& L_{2}=\left(\begin{array}{ll}
0 & 1 \\
0 & 0
\end{array}\right),
\end{aligned}
$$

where $s$ is an arbitrary complex parameter.

In Sec. II, we study the transformation properties of the gauge fields relevant for a doublet of scalar fields transforming locally with the representation (2). In Sec. III, we discuss the elementary properties of these gauge fields and especially the influence of the $s$ parameter. In Sec. IV, we extend our results to all representations where $L_{1}$ can be diagonalized and show in Sec. V how the gauge system of the 2-dimensional representation extends directly to all these representations. In Sec. VI, the Lagrangian of the gauge fields is constructed on general grounds. Finally, we give a brief discussion and conclusions in Sec. VII.

\section{GAUGE TRANSFORMATIONS}

Following step-by-step the procedure pioneered by Yang and Mills, we consider a doublet scalar field in four spacetime dimensions

$$
\Phi(x)=\left(\begin{array}{l}
\Phi_{1}(x) \\
\Phi_{2}(x)
\end{array}\right)
$$

that transforms locally as

$$
\Phi^{\prime}(x)=V(x) \Phi(x)
$$


The infinitesimal form of $V(x)$ is

$$
V(x)=1+\alpha_{1}(x) L_{1}+\alpha_{2}(x) L_{2},
$$

where $L_{1}$ and $L_{2}$ are given by Eq. (2).

The derivative $D_{\mu} \Phi$ is defined by

$$
D_{\mu} \Phi=\left(\partial_{\mu}+A_{\mu}\right) \Phi=\partial_{\mu} \Phi+A_{\mu} \Phi
$$

and must transform in the same way as $\Phi$ itself

$$
\left(D_{\mu} \Phi\right)^{\prime}(x)=V(x)\left(D_{\mu} \Phi\right)(x) .
$$

Equations (4) and (7) imply that

$$
A_{\mu}^{\prime}=V A_{\mu} V^{-1}-\left(\partial_{\mu} V\right) V^{-1},
$$

meaning that this basic result of Yang and Mills is valid for the present gauge group. The infinitesimal form of Eq. (8) is

$$
\begin{aligned}
A_{\mu}^{\prime}= & A_{\mu}+\alpha_{1}\left[L_{1}, A_{\mu}\right]+\alpha_{2}\left[L_{2}, A_{\mu}\right] \\
& -\partial_{\mu} \alpha_{1} L_{1}-\partial_{\mu} \alpha_{2} L_{2}
\end{aligned}
$$

Let us study this equation in some detail. Since $\alpha_{1}$ and $\alpha_{2}$ are arbitrary functions of the space-time variables $x$, this equation implies that there must be at least two gauge fields in $A_{\mu}$. The usual choice is

$$
A_{\mu}(x)=A_{\mu}^{(1)}(x) L_{1}+A_{\mu}^{(2)}(x) L_{2} .
$$

With this choice, which involves two gauge fields $A_{\mu}^{(1)}(x)$ and $A_{\mu}^{(2)}(x)$, Eq. (9) can indeed be satisfied.

Following the discussion of Yang and Mills as quoted in Sec. I, Eq. (10) is not the only possible choice: it is entirely allowed to have more than two gauge fields. As seen from Eq. (6) or Eq. (10) for example, $A_{\mu}(x)$ is a $2 \times 2$ real matrix, and it is therefore natural to consider the case of four gauge fields, namely $A_{\mu}^{i j}, i, j=1,2$. It is convenient to organize these four $A_{\mu}^{i j}$ as a column matrix $A_{\mu}^{a}, a=1,2,3,4$ :

$$
\left(\begin{array}{c}
A_{\mu}^{1} \\
A_{\mu}^{2} \\
A_{\mu}^{3} \\
A_{\mu}^{4}
\end{array}\right) \equiv\left(\begin{array}{c}
A_{\mu}^{11} \\
A_{\mu}^{12} \\
A_{\mu}^{21} \\
A_{\mu}^{22}
\end{array}\right)
$$

In this notation, the transformations of Eq. (9) are

$$
A_{\mu}^{\prime}=A_{\mu}+\alpha_{i} X_{i} A_{\mu}+\partial_{\mu} \alpha_{i} W_{i}
$$

with

$$
X_{1}=\left(\begin{array}{cccc}
0 & 0 & 0 & 0 \\
0 & 1 & 0 & 0 \\
0 & 0 & -1 & 0 \\
0 & 0 & 0 & 0
\end{array}\right) \text {, }
$$

$$
X_{2}=\left(\begin{array}{cccc}
0 & 0 & 1 & 0 \\
-1 & 0 & 0 & 1 \\
0 & 0 & 0 & 0 \\
0 & 0 & -1 & 0
\end{array}\right)
$$

$$
W_{1}=\left(\begin{array}{c}
-\left(\frac{1}{2}+s\right) \\
0 \\
0 \\
\frac{1}{2}-s
\end{array}\right) \text {, }
$$

$$
W_{2}=\left(\begin{array}{c}
0 \\
-1 \\
0 \\
0
\end{array}\right)
$$

The $X_{i}$ of Eqs. (13) and (14) satisfy the same commutation relations (1) of the $L_{1}$ and $L_{2}$, namely,

$$
\left[X_{1}, X_{2}\right]=X_{2} \text {. }
$$

Equations (13)-(16) are very instructive because they exhibit the basic features due to the fact that the gauge group under consideration is not semisimple. These features can be seen as follows. Suppose a linear transformation $T$ is applied to $A_{\mu}$ of Eq. (11) so that the first two components of $T A_{\mu}$ are linear combinations of the gauge fields $A_{\mu}^{(1)}$ and $A_{\mu}^{(2)}$ of Eq. (10). Furthermore, after applying this $T, W_{1}$ and $W_{2}$ both take the form where the third and fourth components are zero. Let $T X_{2} T^{-1}$ be expressed as

$$
T X_{2} T^{-1}=\left(\begin{array}{ll}
Y_{11} & Y_{12} \\
0 & Y_{22}
\end{array}\right),
$$

where the $Y$ 's are $2 \times 2$ matrices. While $Y_{21}$ is zero, the question is: can $Y_{12}$ be made zero or not? Consider any vector with $v_{4} \neq v_{1}$

$$
V_{0}=\left(\begin{array}{c}
v_{1} \\
0 \\
0 \\
v_{4}
\end{array}\right)
$$

which is not a multiple of $W_{1}$. That

$$
X_{2} V_{0}=\left(\begin{array}{c}
0 \\
v_{4}-v_{1} \\
0 \\
0
\end{array}\right)
$$

means that all these $V_{0}$ 's, which do not lie in the space spanned by $W_{1}$ and $W_{2}$, give a $X_{2} V_{0}$ which is proportional to $W_{2}$. This implies that 


$$
Y_{12} \neq 0
$$

That $Y_{12}$ is not zero has profound consequences. If the above considerations are applied to a gauge group that is semisimple, the resulting $Y_{12}$ can always be put to zero. Thus Eq. (21) is a novel feature intimately related to the fact that the present gauge group is not semisimple. Physically, that $Y_{12}$ is nonzero means that the two additional gauge fields are not "irrelevant" and are coupled to the two original gauge fields.

In order to see these new features more clearly, it is convenient to use the following specific linear transform $T$ :

$$
\widetilde{A}_{\mu}^{a}=T^{a b} A_{\mu}^{b}
$$

with

$$
T=\left(\begin{array}{cccc}
0 & 1 & 0 & 0 \\
1 & 0 & 0 & -1 \\
\frac{1}{2}-s & 0 & 0 & \frac{1}{2}+s \\
0 & 0 & 1 & 0
\end{array}\right)
$$

Note that this $T$ is of determinant one and hence invertible whatever the value of $s$ is. One finds that, in this tilde basis, the infinitesimal $\widetilde{A}_{\mu}$ transformation now becomes

$$
\widetilde{A}_{\mu}^{\prime}=\widetilde{A}_{\mu}+\alpha_{i} \widetilde{X}_{i} \tilde{A}_{\mu}+\partial_{\mu} \alpha_{i} \widetilde{W}_{i}
$$

with

$$
\begin{aligned}
& \widetilde{X}_{1}=\left(\begin{array}{cccc}
1 & 0 & 0 & 0 \\
0 & 0 & 0 & 0 \\
0 & 0 & 0 & 0 \\
0 & 0 & 0 & -1
\end{array}\right), \\
& \widetilde{X}_{2}=\left(\begin{array}{cccc}
0 & -1 & 0 & 0 \\
0 & 0 & 0 & 2 \\
0 & 0 & 0 & -2 s \\
0 & 0 & 0 & 0
\end{array}\right), \\
& \widetilde{W}_{1}=\left(\begin{array}{c}
0 \\
-1 \\
0 \\
0
\end{array}\right), \\
& \widetilde{W}_{2}=\left(\begin{array}{c}
-1 \\
0 \\
0 \\
0
\end{array}\right) .
\end{aligned}
$$

With the $T$ of Eq. (23), the $Y_{12}$ of Eq. (18) is explicitly

$$
Y_{12}=\left(\begin{array}{ll}
0 & 0 \\
0 & 2
\end{array}\right)
$$

which is not zero and cannot be brought to zero by any further change of basis respecting Eqs. (27) and (28).

\section{ELEMENTARY PROPERTIES OF GAUGE FIELDS}

In the representation (2) for $L_{1}$ and $L_{2}$, there is a continuous parameter $s$. This is another feature not present for finitedimensional representations of semisimple groups.

In the transformations of the gauge fields $A_{\mu}$ and $\widetilde{A}_{\mu}$ as given by Eqs. (12) and (24) respectively, this parameter $s$ appears explicitly: in $W_{1}$ for $A_{\mu}$ and in $\widetilde{X}_{2}$ for $\widetilde{A}_{\mu}$. Such appearances are undesirable because they imply that both $A_{\mu}$ and $\widetilde{A}_{\mu}$ depend on the representation for the doublet scalar field $\Phi(x)$ of Eq. (3).

We therefore look for a further linear transform of the gauge field such that this $s$ dependence appears in neither the new $X_{i}$ nor the new $W_{i}$. For this purpose, consider first the case

$$
s \neq 0 .
$$

Under this assumption, define similar to Eq. (22),

$$
\hat{A}_{\mu}^{a}=\widetilde{T}^{a b} \widetilde{A}_{\mu}^{b}
$$

with

$$
\widetilde{T}=\left(\begin{array}{cccc}
1 & 0 & 0 & 0 \\
0 & 1 & 0 & 0 \\
0 & 0 & 1 / s & 0 \\
0 & 0 & 0 & 1
\end{array}\right)
$$

The determinant of this diagonal $\widetilde{T}$ is $1 / s$, which is welldefined because of Eq. (30). In the hat basis, the infinitesimal transformation is

$$
\hat{A}_{\mu}^{\prime}=\hat{A}_{\mu}+\alpha_{i} \hat{X}_{i} \hat{A}_{\mu}+\partial_{\mu} \alpha_{i} \hat{W}_{i}
$$

with

$$
\begin{aligned}
& \hat{X}_{1}=\widetilde{X}_{1}=\left(\begin{array}{cccc}
1 & 0 & 0 & 0 \\
0 & 0 & 0 & 0 \\
0 & 0 & 0 & 0 \\
0 & 0 & 0 & -1
\end{array}\right), \\
& \hat{X}_{2}=\left(\begin{array}{cccc}
0 & -1 & 0 & 0 \\
0 & 0 & 0 & 2 \\
0 & 0 & 0 & -2 \\
0 & 0 & 0 & 0
\end{array}\right), \\
& \hat{W}_{1}=\tilde{W}_{1}=\left(\begin{array}{c}
0 \\
-1 \\
0 \\
0
\end{array}\right),
\end{aligned}
$$




$$
\hat{W}_{2}=\widetilde{W}_{2}=\left(\begin{array}{c}
-1 \\
0 \\
0 \\
0
\end{array}\right) \text {. }
$$

There is no dependence on $s$ in Eqs. (34)-(37), as desired. The $Y_{12}$ is still given by Eq. (29).

The $\hat{A}_{\mu}$, not $A_{\mu}$ or $\widetilde{A}_{\mu}$, is the desired gauge field. Let the gauge transform (33) be written out component by component:

$$
\begin{aligned}
& \hat{A}_{\mu}^{1^{\prime}}=\hat{A}_{\mu}^{1}+\alpha_{1} \hat{A}_{\mu}^{1}-\alpha_{2} \hat{A}_{\mu}^{2}-\partial_{\mu} \alpha_{2}, \\
& \hat{A}_{\mu}^{2^{\prime}}=\hat{A}_{\mu}^{2}+2 \alpha_{2} \hat{A}_{\mu}^{4}-\partial_{\mu} \alpha_{1}, \\
& \hat{A}_{\mu}^{3^{\prime}}=\hat{A}_{\mu}^{3}-2 \alpha_{2} \hat{A}_{\mu}^{4}, \\
& \hat{A}_{\mu}^{4^{\prime}}=\hat{A}_{\mu}^{4}-\alpha_{1} \hat{A}_{\mu}^{4} .
\end{aligned}
$$

It is seen from Eqs. (38) and (39) that $\partial_{\mu} \alpha_{2}$ and $\partial_{\mu} \alpha_{1}$ appear respectively in the gauge transforms of $\hat{A}_{\mu}^{1}$ and $\hat{A}_{\mu}^{2}$, similar to those in the original paper of Yang and Mills [1]. We therefore refer to these two components $\hat{A}_{\mu}^{1}$ and $\hat{A}_{\mu}^{2}$ of the gauge fields as Yang-Mills fields of the first kind. In contrast, the derivatives of $\alpha_{1}$ and $\alpha_{2}$ do not appear in the gauge transformations of $\hat{A}_{\mu}^{3}$ and $\hat{A}_{\mu}^{4}$, as given by Eqs. (40) and (41). This is a new feature, and we call these two components $\hat{A}_{\mu}^{3}$ and $\hat{A}_{\mu}^{4}$ of the gauge fields Yang-Mills fields of the second kind.

In this present case of a nonsemisimple gauge group, these two Yang-Mills fields of the second kind are not "irrelevant." As seen from Eq. (39), $\hat{A}_{\mu}^{4}$ appears on the righthand side, and thus plays a role in the gauge transform of the Yang-Mills field $\hat{A}_{\mu}^{2}$ of the first kind. This is the direct consequence of the fact that the $Y_{12}$ of Eq. (18) is not zero.

Aside from a possible linear transform among them, the Yang-Mills gauge fields of the second kind are well defined. When the Yang-Mills fields of the second kind are present, it is allowed to add arbitrary linear combinations of these fields of the second kind to the Yang-Mills gauge fields of the first kind.

It only remains to express the original $A_{\mu}$ in terms of these Yang-Mills fields $\hat{A}_{\mu}$ :

$$
A_{\mu}^{a}=R^{a b} \hat{A}_{\mu}^{b}
$$

with, because of Eq. (30),

$$
R=T^{-1} \widetilde{T}^{-1}=\left(\begin{array}{cccc}
0 & s+\frac{1}{2} & s & 0 \\
1 & 0 & 0 & 0 \\
0 & 0 & 0 & 1 \\
0 & s-\frac{1}{2} & s & 0
\end{array}\right) .
$$

Of course, $A_{\mu}$ of Eq. (42) is to be used in the derivative $D_{\mu} \Phi$ of Eq. (6), and depends on the value of $s$ of the representation for $\Phi$, as expected.

Two comments are appropriate at this point. First, the only transform that leaves $\hat{X}_{1}, \hat{X}_{2}, \hat{W}_{1}$ and $\hat{W}_{2}$ unchanged (sometimes referred as the stability group) is the identity.

Secondly, it is seen from Eq. (43) that the limit $s \rightarrow 0$ is well defined for $R$ itself, and thus it is trivial to remove the restriction (30). In fact,

$$
\left.R\right|_{s \rightarrow 0}=\left(\begin{array}{rrrr}
0 & \frac{1}{2} & 0 & 0 \\
1 & 0 & 0 & 0 \\
0 & 0 & 0 & 1 \\
0 & -\frac{1}{2} & 0 & 0
\end{array}\right) .
$$

In this case of the representation with $s=0$, one Yang-Mills gauge field of the second kind, $\hat{A}_{\mu}^{3}$, decouples.

\section{THE DIAGONAL $L_{1}$ REPRESENTATIONS}

In this section, we describe briefly the nondecomposable representations of our algebra (1) for which $L_{1}$ can be diagonalized. Those of dimension $d=n+1$ are, up to a change of basis, of the form

$$
L_{1}=\left(\begin{array}{ccccc}
\frac{n}{2}+s & 0 & 0 & \ldots & 0 \\
0 & \frac{n}{2}-1+s & 0 & \ldots & 0 \\
0 & 0 & \frac{n}{2}-2+s & \ldots & 0 \\
\vdots & \vdots & \vdots & \ddots & \vdots \\
0 & 0 & 0 & \ldots & -\frac{n}{2}+s
\end{array}\right),
$$

$$
L_{2}=\left(\begin{array}{ccccc}
0 & 1 & 0 & \ldots & 0 \\
0 & 0 & 1 & \ldots & 0 \\
0 & 0 & 0 & \ldots & 0 \\
\vdots & \vdots & \vdots & \ddots & \vdots \\
0 & 0 & 0 & \ldots & 0
\end{array}\right),
$$

where $s$ is an arbitrary complex parameter.

Let us briefly outline the arguments which can be used to prove this result.

Take any representation for which $L_{1}$ is diagonal. The eigenvalues can be classified in sets associated with the following $s_{i}$ 's: 


$$
\begin{array}{ccccc}
s_{1}, & s_{1}+1, & s_{1}+2, & \ldots, & s_{1}+n_{1} \\
s_{2}, & s_{2}+1, & s_{2}+2, & \ldots, & s_{2}+n_{2} \\
\vdots & \vdots & \vdots & \ddots & \vdots \\
s_{i}, & s_{i}+1, & s_{i}+2, & \ldots, & s_{i}+n_{i},
\end{array}
$$

where either $s_{i}-s_{j}$ for $i \neq j$ is not an integer or, if $s_{i}-s_{j}$ is an integer, the corresponding sets are separated by at least 2 units.

Since, for any vector $V$ which is the eigenvector of $L_{1}$ with eigenvalue $s$, we have by the basic commutator relations

$$
L_{1}\left(L_{2} V\right)=\left(L_{2} L_{1}+L_{2}\right) V=(s+1)\left(L_{2} V\right),
$$

we see that two states can be connected by $L_{2}$ only in the case that they belong to one of the above sets (46). Hence, once transformed in the block diagonal form corresponding to the above sets of eigenvalues, the representation decomposes into these blocks. It is sufficient to study each block in turn.

We call $s$ the corresponding lowest eigenvalue and $s+n$ its highest. Suppose that the eigenvalue $s+k$ with $k$ $=0, \ldots, n$ has multiplicity $m_{k} \geqslant 1$.

Take any nonzero vector $V$ eigenstate of $L_{1}$ of eigenvalue $s+k$. The successive action of $L_{2}$ on $V$ generates a set $\left\{S_{V}\right\}$ of nonzero vectors

$$
\begin{aligned}
L_{1} V & =(s+k) V, \\
\left\{S_{V}\right\} & \equiv\left\{V, L_{2} V,\left(L_{2}\right)^{2} V, \ldots,\left(L_{2}\right)^{\mathrm{L}-1} V\right\}, \\
\left(L_{2}\right)^{\mathrm{L}} V & =0, \quad 1 \leqslant \mathrm{~L} \leqslant n-k .
\end{aligned}
$$

Let us call $\mathrm{L}_{V}=\mathrm{L}$ the length of the chain built on $V$.

We now consider the sequence $V_{1}, V_{2}, \ldots$ of vectors defined as follows:

(i) Among all the vectors which are linear combinations of the eigenvectors of $L_{1}$ with eigenvalue $s$, take any vector of minimal length $L_{V_{1}}$ and call it $V_{1}$.

(ii) Among all the vectors which are linear combinations of the eigenvectors of $L_{1}$ with eigenvalue $s$ but not along $V_{1}$, take any vector of minimal length and call it $V_{2}$. Note that the length $L_{V_{2}}$ is larger or equal to the length $L_{V_{1}}$.

(iii) Among all the vectors which are linear combinations of the eigenvectors of $L_{1}$ with eigenvalue $s$ but not situated in the subspace spanned by the vectors $V_{1}, V_{2}$, take any vector of minimal length and call it $V_{3}$.

(iv) Continue the process until the space of eigenvalue $s$ is exhausted, thus defining successively $m_{0}$ vectors.

(v) Among all the vectors which are linear combinations of the eigenvectors of $L_{1}$ with eigenvalue $s+1$ but not situated in the subspace spanned by the vectors $\left\{L_{2} V_{1}, L_{2} V_{2}, \ldots, L_{2} V_{m_{0}}\right\}$, take any vector of minimal length and call it $V_{m_{0}+1}$. If there is no such vector move to the space of eigenvalue $s+2$ and repeat the process. Note that, this time, the length $L_{V_{m_{0}+1}}$ can be larger than the preceding lengths. (vi) Among all the vectors which are linear combinations of the eigenvectors of $L_{1}$ with eigenvalue $s+1$ but not situated in the subspace spanned by the vectors $\left\{L_{2} V_{1}, L_{2} V_{2}, \ldots, L_{2} V_{m_{0}}, V_{m_{0}+1}\right\}$, select a vector of minimal length and call it $V_{m_{0}+2}$. If there is no such vector move to the space of eigenvalue $s+2$ and repeat the process excluding the subspace $\left\{L_{2}^{2} V_{1}, L_{2}^{2} V_{2}, \ldots, L_{2}^{2} V_{m_{0}}, L_{2} V_{m_{0}+1}\right\}$.

(vii) Repeat the process successively for all the eigenvectors of eigenvalues $s+1, s+2, s+3, \ldots$ until the complete space of all eigenvalues of $L_{1}$ is exhausted.

(viii) Remark: This procedure provides a set of vectors

$$
\left\{V_{1}, L_{2} V_{1}, L_{2}^{2} V_{1}, \ldots, V_{2}, L_{2} V_{2}, \ldots\right\}
$$

which are linearly independent. Indeed, if they were not, one would have a combination of vectors of given eigenvalue $s$ $+k$ [see Eqs. (51)] of $L_{1}$ equal to zero:

$$
\begin{aligned}
& \alpha_{p}\left(L_{2}\right)^{a}{ }^{a} V_{p}+\alpha_{q}\left(L_{2}\right)^{a} q V_{q}+\ldots+\alpha_{r}\left(L_{2}\right)^{a} r V_{r} \\
& \quad+\ldots+\left(L_{2}\right)^{a} x V_{x}=0, \\
& \quad \text { for } \quad p<q<\ldots<r<\ldots<x .
\end{aligned}
$$

Note that, if $V_{r}$ corresponds to an eigenvalue $s_{r}$ of $L_{1}$, the integers $a_{p}, a_{q}, \ldots$ obey the relations

$$
\begin{aligned}
& s_{p}+a_{p}=s_{q}+a_{q}=\ldots=s_{r}+a_{r}=\ldots=s_{x}+a_{x}=s+k, \\
& s_{p} \leqslant s_{q} \leqslant \ldots \leqslant s_{r} \leqslant \ldots \leqslant s_{x}, \\
& a_{r}<\mathrm{L}_{V_{r}}, \quad \text { for all } r .
\end{aligned}
$$

By constructing the set $\left\{S_{V_{x}^{\prime}}\right\}$ based on the vector

$$
\begin{aligned}
V_{x}^{\prime}= & \alpha_{p}\left(L_{2}\right)^{a_{p}-a_{x}} V_{p}+\alpha_{q}\left(L_{2}\right)^{a} q^{-a_{x}} V_{q} \\
& +\alpha_{r}\left(L_{2}\right)^{a_{r}-a_{x}} V_{r}+\ldots+V_{x}, \\
L_{2}^{a_{x}} V_{x}^{\prime}= & 0
\end{aligned}
$$

rather than $V_{x}$, one would construct the vector $V_{x}^{\prime}$ of length $a_{x}$ smaller than the length of $V_{x}$ [see Eqs. (51)] contrary to the hypothesis.

(ix) To any vector in the constructed series $V_{r}$, there corresponds the set $\left\{S_{V_{r}}\right\}$, the basis of a nondecomposable representation of the group of the form (45) with a dimension $d=\mathrm{L}_{V_{r}}$ and with a well-chosen

$$
s=s_{r}+\frac{L_{V_{r}}-1}{2} .
$$

This ends the proof of the decomposition of the representations for which $L_{1}$ is diagonal.

We end this section with a word on the nondecomposable representations where $L_{1}$ assumes a nondiagonal form, in fact a Jordan form. There are many such representations. Some have a very elaborate structure. It is sufficient for our 
later purpose to write the simplest example which is 3-dimensional and assumes the form

$$
\begin{aligned}
L_{1} & =\left(\begin{array}{ccc}
\frac{2}{3}+s & 0 & 0 \\
0 & -\frac{1}{3}+s & 1 \\
0 & 0 & -\frac{1}{3}+s
\end{array}\right), \\
L_{2} & =\left(\begin{array}{lll}
0 & 0 & 1 \\
0 & 0 & 0 \\
0 & 0 & 0
\end{array}\right) .
\end{aligned}
$$

\section{GAUGE FIELDS FOR MATTER FIELDS BELONGING TO A DIAGONAL $L_{1}$ REPRESENTATION}

In this section, we give the arguments showing that, for a matter field belonging to a general diagonal $L_{1}$, nondecomposable, $d$-dimensional representation, the gauge field structure is exactly the same as for a matter field transforming with the two-dimensional representation (2). It consists of two gauge fields of the first kind and two gauge fields of the second kind. There are $d^{2}-4$ other gauge fields which are "irrelevant" as they decouple from the gauge fields of the first and the second kinds.

Suppose that the matter field $\Phi(x)$ has $d$ scalar components and transforms as in Eqs. (4),(5) with the infinitesimal $L_{1}, L_{2}$ given by Eq. (45). In the generalized derivatives there appears a set of four $d \times d$ matrices $A_{\mu}$ transforming as Eqs. (8),(9). It is again convenient to associate the matrix $A_{\mu}$, with components $A_{\mu}^{i j}, i, j=1, \ldots, d$, with the $d^{2}$-dimensional vector $A_{\mu}^{a}, a=1, \ldots, d^{2}$

$$
A_{\mu} \Rightarrow A_{\mu}^{a}
$$

by

$$
A_{\mu}^{d(i-1)+j}=A_{\mu}^{i j}
$$

For these $A_{\mu}^{a}$ fields, the gauge transformations are again of the form (12). The $X_{i}$ are $d^{2} \times d^{2}$ matrices generalizing Eqs. (13) and (14). The two $d^{2}$-dimensional vectors $W_{1}$ and $W_{2}$ are those associated with $-L_{2}$ and $-L_{1}$, respectively. It is however somewhat easier to continue to work with the matrix $A_{\mu}^{i j}$, using the initial $L_{1}$ and $L_{2}$ matrices (45) and the commutator action (9).

Let us introduce the following four $d \times d$ matrices, $M_{m}, m=-1,0,1$ and $P_{0}$, and thus the corresponding vectors. The nonzero elements of these matrices are

$$
\begin{array}{r}
M_{-1}(j+1, j)=-\frac{(d-j) j}{2}, \quad j=1, \ldots, d-1, \\
M_{0}(j, j)=-\frac{d-2 j+1}{2}, \quad j=1, \ldots, d,
\end{array}
$$

$$
\begin{gathered}
M_{1}(j, j+1)=1, \quad j=1, \ldots, d-1, \\
P_{0}(j, j)=1, \quad j=1, \ldots, d .
\end{gathered}
$$

These matrices obey commutation rules with $L_{1}$ and $L_{2}$ which govern the homogeneous part of the transformation rules of the gauge vectors [see Eq. (9)]:

(i) The three $M_{m}$ transform infinitesimally as a 3-dimensional representation of our algebra

$$
\begin{aligned}
& {\left[L_{1}, M_{m}\right]=m M_{m},} \\
& {\left[L_{2}, M_{1}\right]=0,} \\
& {\left[L_{2}, M_{m}\right]=M_{m+1}, \quad m=-1,0 .}
\end{aligned}
$$

(ii) The last one, $P_{0}$, transforms as the 1-dimensional representation

$$
\begin{aligned}
& {\left[L_{1}, P_{0}\right]=0,} \\
& {\left[L_{2}, P_{0}\right]=0 .}
\end{aligned}
$$

Finally, we have the correspondence

$$
\begin{aligned}
& L_{1}=-M_{0}+s P_{0} \Rightarrow-W_{2}, \\
& L_{2}=M_{1} \Rightarrow-W_{1} .
\end{aligned}
$$

The gauge vectors which lie in the direction of these four vectors are the four "relevant" gauge fields. They contain the gauge fields of the first kind in the directions of $W_{1}$ and $W_{2}$ and the two gauge fields of the second kind. The $d^{2}-4$ remaining vectors can be classified by using the reduction of a general representation as described in Sec. IV starting the procedure with the vector $V_{1}$ of the lowest value of $s$ $=-(d-1) / 2$ corresponding to the matrix with only one nonzero element, namely, $V_{1}(d, 1)=1$.

Using the notation $[d]$ for a representation of dimension $d$, it is easy to see that the resulting decomposition of the action on the $d^{2}$ gauge fields (56) is as follows:

$$
\left[d^{2}\right]=\sum_{\oplus i=0}^{d-1}[2 i+1]=[1] \oplus[3] \oplus \sum_{\oplus i=2}^{d-1}[2 i+1],
$$

where the part $[4]=[1] \oplus[3]$ is equivalent to Eqs. (57) and, in the basis just referred to, acts on the above 4-dimensional space which contains the subspace spanned by vectors $W_{1}$ and $W_{2}$. The other $(2 i+1)$-dimensional representations (with $2 \leqslant i \leqslant d-1$ ) are the nondecomposable ones of the diagonal case [Eq. (45) with their own $\left.s_{i}=0\right]$.

In a way completely analogous to the argument outlined around Eq. (18), we apply a transformation $T$ in such a way as to bring the linear combinations of the four vectors (57) in positions $1,2,3,4$ and to put the $d^{2}-4$ other vectors obtained in the procedure in the remaining positions $5, \ldots, d^{2}$. Then, both the $d^{2} \times d^{2}$ matrices $X_{1}$ and $X_{2}$ take the form 


$$
T X_{i} T^{-1}=\left(\begin{array}{ll}
Z_{11}^{i} & 0 \\
0 & Z_{22}^{i}
\end{array}\right),
$$

where $Z_{11}^{i}$ is a $4 \times 4$ matrix analogous to Eq. (18) and $Z_{22}^{i}$ a $\left(d^{2}-4\right) \times\left(d^{2}-4\right)$ matrix. The nondiagonal blocks are zero.

Hence we conclude that all matter fields transforming with diagonal representations belong to the same theory with, in the general case, exactly the same four vector fields (57). Again there are two Yang-Mills gauge fields of the first kind and two Yang-Mills gauge fields of the second kind. The gauge fields corresponding to the $d^{2}-4$ remaining vectors are "irrelevant" as seen from Eq. (62).

To conclude this section let us write explicitly the matrix $A_{\mu}$ which has to be used in the covariant derivative (6) of the $d$-dimensional field

$$
\begin{aligned}
A_{\mu} & =\hat{A}_{\mu}^{1} M_{1}-\hat{A}_{\mu}^{2} M_{0}-2 \hat{A}_{\mu}^{4} M_{1}+s\left(\hat{A}_{\mu}^{2}+\hat{A}_{\mu}^{3}\right) P_{0} \\
& =\hat{A}_{\mu}^{2} L_{1}+\hat{A}_{\mu}^{1} L_{2}+s \hat{A}_{\mu}^{3} P_{0}-2 \hat{A}_{\mu}^{4} M_{1} .
\end{aligned}
$$

Again we see that for $s=0$, the field $\hat{A}_{\mu}^{3}$ decouples.

From Eq. (63), we see the correspondence between the two Yang-Mills gauge fields usually called $A_{\mu}^{(1)}$ and $A_{\mu}^{(2)}[$ see Eq. (10)] and our hat fields $\hat{A}_{\mu}^{1}$ and $\hat{A}_{\mu}^{2}$ :

$$
\begin{aligned}
& A_{\mu}^{(1)}=\hat{A}_{\mu}^{2}, \\
& A_{\mu}^{(2)}=\hat{A}_{\mu}^{1} .
\end{aligned}
$$

If an analogous study is performed using, for the scalar fields, another nondecomposable representation with a nondiagonal $L_{1}$, the picture changes drastically. We have analyzed in full detail what happens for a few of these representations and in particular for the representation (54). In the latter case there are, apart from the two Yang-Mills fields of the first kind, in general seven Yang-Mills fields of the second kind. This new system of altogether nine Yang-Mills fields does not contain the set of the four Yang-Mills fields relevant to the representations where $L_{1}$ is diagonal.

\section{THE GAUGE FIELD LAGRANGIAN}

We can write easily a gauge invariant Lagrangian which is, up to a factor,

$$
\mathcal{L}^{I}=\operatorname{trace}\left(F^{\mu \nu} F_{\mu \nu}\right)
$$

familiar for simple groups but also

$$
\mathcal{L}^{I I}=\left(\operatorname{trace} F^{\mu \nu}\right)\left(\operatorname{trace} F_{\mu \nu}\right) \text {. }
$$

These results are obvious if the curvatures are defined, as usual, by

$$
F_{\mu \nu}=\left[D_{\mu}, D_{\nu}\right]
$$

which, in view of Eq. (7), transform as

$$
\begin{aligned}
\left(F_{\mu \nu}\right)^{\prime} & =V F_{\mu \nu} V^{-1} \\
& =F_{\mu \nu}+\alpha_{i}\left[X_{i}, F_{\mu \nu}\right] \quad \text { infinitesimally. }
\end{aligned}
$$

This means that the curvatures are invariant under the nonhomogeneous part of the transformation (24) induced by the $W$ 's and transform covariantly under the homogenous part induced by $V$. The invariance of the Lagrangians (65) and (66) follows. It should be noted that these Lagrangians are invariant not only under our gauge group but more generally under transformations with any matrix $V$, namely the group $\mathrm{GL}(2, R)$ or even $\mathrm{GL}(2, C)$, which contain our group as a subgroup.

Since the $\hat{A}_{\mu}^{a}, a=1, \ldots, 4$ gauge fields are the basic fields of the first and of the second kind for all the diagonal representations, we focus our attention on them. Recall that their infinitesimal transformation properties are summarized in Eq. (33) with $X_{i}$ and $W_{i}$ given by Eqs. (34)-(37).

In view of the new feature related to the presence of gauge fields of the second kind, we did not want to be prejudiced by the familiar result and we have decided to start with a minimal set of general conditions.

(i) The Lagrangian should be Lorentz invariant.

(ii) Terms of a kinetic energy type for the vector fields should appear in the Lagrangian, i.e., a sum of terms quadratic in the space-time derivatives of the fields

$$
\beta_{1}(a, b)\left(\partial_{\mu} \hat{A}_{\nu}^{a}\right)\left(\partial^{\mu} \hat{A}^{b \nu}\right)+\beta_{2}(a, b)\left(\partial_{\nu} \hat{A}_{\mu}^{a}\right)\left(\partial^{\mu} \hat{A}^{b \nu}\right)
$$

for a suitable set of values of the constants $\beta_{i}(a, b)$.

(iii) The Lagrangian should be invariant under the gauge transformations.

(iv) Terms which are total divergences can be eliminated.

As a result of condition (iii) and because of the existence of the inhomogeneous part in the transformation, two types of terms should be added to the expression (69): (i) terms quadratic in the fields and at the same time linear in the space-time derivatives

$$
\gamma(a, b, c)\left(\partial_{\nu} \hat{A}_{\mu}^{a}\right) \hat{A}^{b \nu} \hat{A}^{c \mu}
$$

(ii) terms quartic in the vector fields

$$
\delta(a, b, c, d) \hat{A}_{\mu}^{a} \hat{A}^{b \mu} \hat{A}_{\nu}^{c} \hat{A}^{d \nu}
$$

The coefficients $\beta_{1}(a, b), \quad \beta_{2}(a, b), \quad \gamma(a, b, c)$ and $\delta(a, b, c, d)$ obviously enjoy the symmetries

$$
\begin{gathered}
\beta_{1}(a, b)=\beta_{1}(b, a), \\
\beta_{2}(a, b)=\beta_{2}(b, a), \\
\delta(a, b, c, d)=\delta(b, a, c, d), \\
\delta(a, b, c, d)=\delta(a, b, d, c), \\
\delta(a, b, c, d)=\delta(c, d, a, b) .
\end{gathered}
$$


Taking the most general linear combination of terms of the form (69), (70) and (71), after lengthy computations, we have shown that we recover, in general, a linear combination of the two obvious Lagrangians (65) and (66) and nothing more.

More precisely (i) first as a consequence of the space dependence of the parameters, i.e. to the presence of the inhomogeneous terms in the transformation, we have obtained the expected result: the Lagrangian can be written in terms of the covariant derivatives $\hat{F}^{\mu \nu a}$ only. The Lagrangian then takes the form

$$
\beta_{a b} \hat{F}^{\mu \nu a} \hat{F}_{\mu \nu}^{b}
$$

(ii) Global symmetry then remains to be imposed. This leads to the final restrictions

$$
\begin{aligned}
& \beta_{11}=\beta_{12}=\beta_{13}=\beta_{24}=\beta_{34}=\beta_{44}=0, \\
& \beta_{14}=2\left(\beta_{22}-\beta_{33}\right), \\
& \beta_{23}=\beta_{33} .
\end{aligned}
$$

We see that there are two free parameters $g_{1}=\beta_{22}$ and $g_{2}$ $=\beta_{33}$.

The final form of the most general invariant Lagrangian in the hat basis is then

$$
\begin{aligned}
\mathcal{L}= & g_{1}\left(\hat{F}^{(2) \mu \nu} \hat{F}_{\mu \nu}^{(2)}+4 \hat{F}^{(1) \mu \nu} \hat{F}_{\mu \nu}^{(4)}\right)+g_{2}\left(\hat{F}^{(3) \mu \nu} \hat{F}_{\mu \nu}^{(3)}\right. \\
& \left.+2 \hat{F}^{(2) \mu \nu} \hat{F}_{\mu \nu}^{(3)}-4 \hat{F}^{(1) \mu \nu} \hat{F}_{\mu \nu}^{(4)}\right) .
\end{aligned}
$$

Going back from the hat basis to the initial basis for the $A_{\mu}$ 's by the $R$ transform of Eq. (42), we recover for the particular values

$$
g_{1}=\frac{5}{2}, \quad g_{2}=2
$$

the usual Lagrangian (65) as a particular case, while for

$$
g_{1}=g_{2}=4
$$

we recover the second Lagrangian (66).

We defer to a later work the study of the possible physical consequences of the fact that, by choosing suitably $g_{1}$ and $g_{2}$, certain gauge fields could have no kinetic energy (for example if $g_{2}=0$ ) and hence can be eliminated from the equation of motion though they appear explicitly in the Lagrangian.

\section{CONCLUSIONS AND DISCUSSION}

As shown almost 50 years ago by Yang and Mills [1] when they discovered and introduced gauge theories, the number of gauge fields can always be chosen to be equal to the number of the generators of the gauge group. This result is valid for any group.

Moreover, Yang and Mills already discussed in their original article the possibility of additional gauge fields. They remarked that, for semisimple groups and semisimple groups multiplied by U(1)'s, these additional gauge fields can be removed from the theory because they are irrelevant. A way of understanding their idea is to note that, for these groups, the gauge transformations of the additional fields completely decouple from the original Yang-Mills fields. In other words, Yang-Mills fields transform among themselves and the additional gauge fields among themselves separately.

In this paper, we address the issue of these additional gauge fields for nonsemisimple groups. It is found that, for this case as distinct from that of the semisimple groups possibly multiplied by U(1)'s, there can be additional gauge fields that are not irrelevant. That is, these additional gauge fields appear in the gauge transform of the original YangMills fields. In such cases, we refer, by definition, to the original Yang-Mills fields as Yang-Mills gauge fields of the first kind, and to the additional Yang-Mills fields as YangMills gauge fields of the second kind. Yang-Mills gauge fields of the second kind are well defined, but it is permitted to alter Yang-Mills gauge fields of the first kind by adding to them arbitrary linear combinations of Yang-Mills gauge fields of the second kind.

The case of the simplest nonsemisimple group, where there are only two generators $L_{1}$ and $L_{2}$ which satisfy Eq. (1), is worked out in detail. In this case we have studied and determined explicitly what happens when the matter field transforms as a representation of that group for which $L_{1}$ can be diagonalized. Apart from the two Yang-Mills gauge fields of the first kind corresponding to the two generators, the allowed additional gauge fields separate into two Yang-Mills gauge fields of the second kind with the remaining gauge fields being irrelevant. No change of basis allows the decoupling of the gauge fields of the second kind and hence the elimination of these additional gauge fields.

For matter fields belonging to representations of our group where $L_{1}$ cannot be diagonalized, the situation is much more complicated. For only one of these cases are the results reported briefly in this article.

We have shown that the Lagrangian for the gauge fields is not unique since the most general gauge invariant Lagrangian depends on two arbitrary parameters. For certain values of the parameters, some gauge fields may have no kinetic energy and appear in the theory as nonpropagating fields of spin 1.

A general argument can be given as follows to indicate that the behavior we have discovered for the above simplest group is generic. For semisimple groups, the reducible representations are all fully reducible (or decomposable). This is not true for nonsemisimple groups. Since the gauge fields belong to the direct product of the representation of the matter field with its inverse transposed, this product is always reducible as it contains the adjoined representation to which the Yang-Mills gauge fields of the first kind belong. But since for nonsemisimple groups this product is in general reducible but not fully reducible, there will be Yang-Mills gauge fields of the second kind connected to the Yang-Mills fields of the first kind, in a way analogous, with appropriate changes, to Eqs. (18) and (21). 


\section{ACKNOWLEDGMENTS}

A large part of this work was carried out while the authors were visiting CERN. We are very grateful to the Theory Division for its hospitality. The work of one of the authors

[1] C.N. Yang and R.L. Mills, Phys. Rev. 96, 191 (1954).

[2] S. Glashow, Nucl. Phys. 22, 579 (1961).

[3] S. Weinberg, Phys. Rev. Lett. 19, 1264 (1967).
(J.N.) was supported in part by the Belgian Fonds National de la Recherche Scientifique, while that of the other (T.T.W.) was supported in part by the United States Department of Energy under Grant No. DE-FG02-84ER40158.

[4] A. Salam, in Elementary Particle Theory: Relativistic Groups and Analyticity (Nobel Symposium No. 8), edited by N. Svartholm (Almqvist and Wiksell, Stockholm, 1968). 